\title{
THE BEST APPROXIMATION AND AN EXTENSION OF A FIXED POINT THEOREM OF F.E. BROWDER
}

\author{
V.M. SEHGAL' and S.P. SINGH² \\ ${ }^{1}$ Dcpartment of Mathematics, Unzversity of Wyoming, Larame, WY', 8207I, U.S.A. \\ ${ }^{2}$ Department of Mathematıcs and Statıstıcs, Memorial Untversity of Newfoundland, St. John's, \\ Newfoundland, Canada, $A 1 C^{\prime} 5 S Y$
}

(Received March 17, 1994 and in revised form May 9, 1994)

\begin{abstract}
In this paper, the KKM principle has been used to obtain a theorem on the best approximation of a continuous function with respect to an affine map. The main result provides extensions of some well-known fixed point theorems.
\end{abstract}

KEY WORDS AND PHRASES. Best approximation, fixed point theorem, KKM-map, p-affine map, inward set.

\section{MATHEMATICS SUBJECT CLASSIFICATIONS: Primary 47H10, Secondary 54II25.}

Let $E$ be a locally convex topological vector space and $C$ a non-empty subset of $L$. A mapping $p:(r \times b \rightarrow[0, \infty)$ is a convex map iff for each fixed $x \in C, p(r, \cdot): E \rightarrow(0, \infty)$ is a convex function. For $x \in C$, the inward set $I_{C}(x)=\left\{x+r(y-x): y \in C^{\prime}, r>0\right\}$. Browder [1] proved the following extension of the Schauder's fixed point theorem.

THEOREM 1. (Browder). Let $C$ be a compact, convex subset of $E$ and $\int:(; \rightarrow b)$ a continuous map. If $p: C \times E \rightarrow[0, \infty)$ is a continuous convex map satisfying

(1) for each $x \neq f(x)$, there exists a $y \in I_{C}(x)$ with $p(x, f(x)-y)<p(x, f(x)-x)$, then $\int$ has a fixed point.

It may be stated that the importance of Theorem 1 stems from $p$ being a continuous convex map instead of a continuous seminorm on $E$. In this paper, we use the KKM principle to obtain a result on the 'best approximation' that yields Theorem 1 with relaxed hypothesis on compactness.

Let $X$ be a non-empty subset of $E$. Recall that a mapping $F: X \rightarrow 2^{E}$ is a KKM map if $F(x) \neq \emptyset$ for each $x \in X$, and for any finite subset $A=\left\{x_{1}, x_{2}, \ldots, x_{n}\right\} \subseteq X, C_{0}(A) \subseteq U\left\{F^{\prime}\left(r_{1}\right)\right.$ : $i=1,2, \ldots, n\}$, where $C_{0}(A)$ denotes the convex hull of $A$. Observe that if $F$ is a KKM map, then $x \in F(x)$ for each $x \in X$.

It is shown by Fan [2] that if $F: X \rightarrow 2^{E}$ is a closed valued KKM map, then the family $\{F(x): x \in X\}$ has the finite intersection property.

As an immediate consequence of the above result, we have:

LEMMA 2. If $X$ is a non-empty compact, convex subset of $E$ and $F: X \rightarrow 2^{E}$ is a closed valued KKM map, then $\cap\{F(x): x \in X\} \neq \emptyset$. 
PROOF. Define a map $G: X \rightarrow 2^{X}$ by

$$
C^{\prime}(x)=l^{\prime}(x) \cap X .
$$

Then $G(x)$ is a nonempty compact subset of $X$ and $(;$ is a KKM map. Consequently, by [2], $\{G(x): x \in X\}$ has the finite intersection property. Since $X$ is compact, it follows that $\cap\{(i($.$) :$ $x \in X\} \neq \emptyset$, and hence, $\cap\{F(x): x \in X\} \neq \emptyset$.

The following lemma is essentially due to Kim [3]. We give a proof for completeness.

Note: In the following, $C_{0}(A)$ : stands for the closed convex hull of $A$.

LEMMA 3. If $A$ and $B$ are compact, convex subsets of $E$, then $C_{0}(A \cup B)$ is a commpart, convex subset of $E$.

PROOF. Since $A$ and $B$ are convex, it follows $C_{0}(A \cup B)=\{\lambda x+\mu y: x \in A, y \in$ $B, \lambda, \mu \in[0,1]$ and $\lambda+\mu=1\}$. Clearly, $C_{0}(A \cup B)$ is a closed and convex subset of $E$. To show that $C_{0}(A \cup B)$ is compact,let $C=[0,1] \times[0,1] \times A \times B$ and $D=\{\lambda x+\mu y: x \in A, y \in B, \lambda, \mu \in|0,1|\}$. Then $C$ is a compact subset of $Y=[0,1] \times[0,1] \times E \times E$ in the product topology on $Y$. Further, the mapping $f: Y \rightarrow E$ defined by $f(\lambda, \mu, x, y)=\lambda x+\mu y$ being continuous, it follows that $I)=f\left(\left(^{\prime}\right)\right.$ is a compact subset of $E$ and, hence, $C_{0}(A \cup B) \subseteq D$ is compact.

LEMMA 4. Let $X$ be a non-empty convex subset of $E$ and $F: X \rightarrow 2^{E}$ a closed ralued likM map. If there exists a compact, convex set $S \subseteq X$ such that $\cap\left\{F(x): x \in S^{\prime}\right\}$ is non-('mply and compact, then $\cap\{F(x): x \in X\} \neq \emptyset$.

PROOF. Let $C=\cap\{F(x): x \in S\}$. Then $C$ is non-empty and a compact subset of $E$. To prove the lemma, it suffices to show that the family $\{F(x) \cap C: x \in X\}$ has the finite intersection property. To prove this, let $A$ be a finite subset of $X$. Then $C_{0}(A)$ is compact and by Lemma 3, $D=C_{0}\left(S \cup C_{0}(A)\right)$ is a compact and convex subset of $X$. Consequently, by Lemma 2, $\cap\{F(x): x \in D\} \neq \emptyset$. This implies that $\cap\{F(x) \cap C: x \in A\} \neq \emptyset$. Thus, $\left\{r(x) \cap C^{\prime}: x \in X\right\}$ has the finite intersection property. Since $C$ is compact and $F(x)$ is closed for each $x \in X$, it follows that $\cap\{F(x) \cap C: x \in X\} \neq \emptyset$. This implies that $\cap\{F(x): x \in X\} \neq \emptyset$.

Let $X$ be a non-empty convex subset of $E$ and $p: X \times E \rightarrow[0, \infty)$ a convex map. $\Lambda$ mapping $g: X \rightarrow X$ is a $p$-affine map iff for each triple $\left\{x, x_{1}, x_{2}\right\} \subseteq X, y \in E$, and $\lambda, \mu \in[0,1]$ with $\lambda+\mu=1$,

$$
p\left(x, y-g\left(\lambda x_{1}+\mu x_{2}\right)\right) \leq \max \left\{p\left(x, y-g\left(x_{\imath}\right)\right): i=1,2\right\} .
$$

Note: If $g$ is linear or affine in the sense of Prolla [4], then $p$ being convex, it follows that $g$ is $p$-afline in the above sense. It is immediate that if $g$ is $p$-affine, then for any finite set $A=\left\{x_{1}, x_{2}, \ldots, x_{n}\right\} \subseteq$ $X$ and $\lambda_{i} \geq 0$ with $\sum_{i=1}^{n} \lambda_{i}=1$,

$$
p\left(x, y-g\left(\sum_{i=1}^{n} \lambda_{\imath} x_{\imath}\right)\right) \leq \max \left\{p\left(x, y-g\left(x_{i}\right)\right): i=1,2, \ldots, n\right\}
$$

for each $x \in X, y \in E$.

The following is the main result of this paper.

THEOREM 5. Let $X$ be a nonempty convex subset of $E$ and $p: X \times E \rightarrow[0, \infty)$ a continuous convex map. Let $f: X \rightarrow E$ and $g: X \rightarrow X$ be continuous mappings with $g$-affinc. Suppose there exist a compact, convex set $S \subseteq X$ and a compact set $K \subseteq X$ such that

(2) for each $y \in X \backslash K$ there exists an $x \in S$ such that $p(y, f(y)-g(y))>p(y, f(y)-g(x))$. T'hen there exists a $u \in X$ that satisfies

(3) $p(u, f(u)-g(u))=\inf \{p(u, f(u)-g(x)): x \in X\}=\inf \left\{p(u, f(y)-z): z \in \operatorname{cl}_{X}(g(u))\right\}$.

PROOF. We first prove the left equality. For this, we define a mapping $G: X \rightarrow 2^{X}$ by

$$
G(x)=\{y \in X: p(y, f(y)-g(y)) \leq p(y, f(y)-g(x))\} .
$$


Clearly, $x \in G^{\prime}(x)$ and it follows that $G^{\prime}(x)$ is closed for each $x \in X$. We show that $('$ is a KKM map. Let $y=\sum_{t=1}^{n} \lambda_{t}, r_{1}, \lambda_{1} \geq 0, \sum_{t=1}^{n} \lambda_{t}=1, x_{t} \in X$ for each 2 . Suppose $y \notin \cup\left\{\left(i\left(x_{t}\right), \imath=1,2, \ldots, n\right\}\right.$. Then for each $\imath=1,2, \ldots, n$,

$$
p(y, f(y)-g(y))>p\left(y, f(y)-g\left(x_{1}\right)\right) .
$$

This implies that $p(y, f(y)-g(y))=p\left(y, f(y)-g\left(\sum_{t=1}^{n} \lambda_{1} x_{1}\right)\right) \leq \max \left\{p\left(y, f(y)-y\left(x_{1}\right)\right), 1=\right.$ $1,2, \ldots, n\}<p(y, f(y)-g(y))$. This inequality is impossible and, consequently, $y \in U\left\{\left(i\left(r_{1}\right)\right.\right.$ : $i=1,2, \ldots, n\}$, that is, $G$ is a closed valued map. Now, since $S$ is a compact convex subset of $X$, it follows by Lemma 2 that $C=\cap\{G(x): x \in S\}$ is a nonempty closed subset of $X$. We show that $C \subseteq K$. Suppose $y \in C$ and assume that $y \in X \backslash K$. Then by hypothesis there exists an $x \in S$ such that $p(y, f(y)-y(y))>\eta(y, f(y)-g(x))$. This implies that $y \notin(i(r)$ for an $x \in S$ and, hence, $y \notin C$, contradicting the initial supposition. Thus, (' $\subseteq K$ and, hence, $\cap\{G(x): x \in S\}$ is a nonempty compact subset of $K$. Hence by Lemma $4, \cap\{(i(x): x \in X\} \neq \phi$. If $u \in \cap\{G(x): x \in X\}$, then for each $x \in X, p(u, f(u)-g(u)) \leq p(u, f(u)-g(x))$. Further, since $u \in X$, it follows that $p(u, f(u)-g(u))=\inf \{p(u, f(u)-g(x)): x \in X\}$. This proves the first equality in (3). To prove right side of the equality in (3) we first show that for each $z \in I_{X}(g(u)) \backslash X, p(u, f(u)-g(u)) \leq p(u, f(u)-z)$. Now $z \in I_{X}(g(u)) \backslash X$ implies that there is a $y \in X$ and $r>1$ such that $y=\frac{1}{r} z+\left(1-\frac{1}{r}\right) g(u)$. Hence, by the first equality and $p$ being convex, it follows that $p(u, f(u)-g(u)) \leq p(u, f(u)-y) \leq \frac{1}{r} p(u, f(u)-z)+\left(1-\frac{1}{r}\right) p(u, f(u)-q(u))$, that is, $p(u, f(u)-g(u)) \leq p(u, f(u)-z)$ for each $z \in I_{X}(g(u)) \backslash X$. Since the last inequality is also true for any $z \in X$, it follows that $p\left(u, \int(u)-g(u)\right) \leq p(u, f(u)-z)$ for each $z \in I_{X}(\eta(u))$. Further, since the functions $f, g$, and $p$ are continuous and $g(u) \in I_{X}(g(u))$, it follows that $p(u, f(u)-g(u))=\inf \left\{p(u, f(u)-z): z \in \operatorname{cl}\left(I_{X}(g(u))\right)\right\}$. This proves the second equality in (3).

As a simple consequence of Theorem 2, we have

COROLLARY 6. Suppose $X$ is a compact, convex subset of $E, p: X \times l i \rightarrow[0, \infty)$ a contrnuous convex function and $f: X \rightarrow E$ a contmuous function. Then for any conflnuous p-affine map $g: X \rightarrow X$, there exists a $u \in X$ that satisfies (3). Further,

(i) if $f(x) \in \operatorname{cl}\left(I_{X}(g(x))\right)$ for cach $x \in X$ then $p(u, f(u)-g(u))=0$,

(ii) if for each $x \in X$, with $f(x) \neq g(x)$ there exists a $y \in \operatorname{cl}\left(I_{X}(g(x))\right)$ such that $p(x, f(x)-y)<$ $p(x, f(x)-g(x))$, then $f(u)=g(u)$.

PROOF. Set $S=K=X$ in Theorem 5. Since $X \backslash K=\phi$, condition (2) in Theorem 5 is satisfied. Hence, there is a $u \in X$, that satisfies (3). Clearly, (i) implies $p(u, f(u)-g(u))=0$. To prove (ii), suppose $f(u) \neq g(u)$. Then by hypothesis $p(u, f(u)-z)<p(u, f(u)-g(u))$ for some $z \in \operatorname{cl}\left(I_{X}(g(u))\right)$. The last inequality contradicts (3). Hence, $f(u)=g(u)$.

It may be remarked that if $g$ is the identity mapping of $X$, then Corollary 6 yields Browder's Theorem 1 and also extends a recent result of Sehgal, Singh, and Gastl [5] if $f$ therein is a single valued map.

For the next result, let $\boldsymbol{P}$ denote the family of nonnegative continuous convex functions on $X \times E$. Note if $p_{1}$ and $p_{2} \in \boldsymbol{P}$, then so is $p_{1}+p_{2}$. Also, if $p$ is a continuous seminorm on $E$, then $p$ generates a nonnegative continuous convex function on $X \times E$ defined by $\hat{p}(x, y)=p(y)$. A mapping $g: X \rightarrow X$ is $\boldsymbol{P}$ affine if it is $p$-affine for each $p \in \boldsymbol{P}$.

The result below is an extension of an earlier result of Fan.

THEOREM 7. Let $X$ be a compact, convex subset of $E$ and $f: X \rightarrow E$ a conlıuuous function. Then for any contınuous $\boldsymbol{P}$ affine map $g: X \rightarrow X$,

(4) etther $f(u)=g(u)$ for some $u \in X$, 
(5) or there cxists $a p \in \boldsymbol{P}$ and $a u \in X$ with $0<p(u, f(u)-g(u))=\inf \{p(u, f(u)-z): z \in$ $c l\left(I_{X}(g(u))\right)$.

In partacular, if $f(x) \in \operatorname{cl}\left(I_{X}(g(x))\right)$ for cach $x$, then (5) holds.

PROOF. It follows by Theorem 5 that for each $p \in \boldsymbol{P}$ there is a $u=u_{p} \in \mathcal{X}$ such that $p(u, f u-g u)=\inf \left\{p(u, f(u)-z): z \in \operatorname{cl}\left(I_{X}(g(u))\right)\right\}$. If for some $p, p(u, f(u)-g(u))>0$, then (5) is true. Suppose then, $p\left(u_{p}, f\left(u_{p}\right)-g\left(u_{p}\right)\right)=0$ for each $p \in \boldsymbol{P}$. Set $A_{p}=\{u \in X: p(u, f(u)-v(u))=$ $0\}$. Then $A_{p}$ is a nonempty compact subset of $X$. Furthermore, the family $\left\{A_{P}: p \in \boldsymbol{P}\right\}$ has the finite intersection property. Consequently, there is a $u \in X$ that satisfies

(6) $p(u, f(u)-g(u))=0$ for each $p \in \boldsymbol{P}$.

If $f(u) \neq g(u)$, then since $E$ is separated, there exists a continuous seminorm $p$ on $E$ such that $p(f(u)-g(u)) \neq 0$ and, hence, $\hat{p}(u, f(u)-g(u))>0$, contradicting (6). Thus, $f(u)=g(u)$. IIence, (5) holds in the alternate case.

\section{REFERENCES}

BROWDER, F.E. On a sharpened form of Schauder fixed point theorem, Proc. Natl. Acad. Sci., U.S.A., 76 (1977), 4749-4751.

FAN, K. Extensions of two fixed point theorems of F.E. Browder, Math. Z, 112 (1969), 234-240.

KIM, W.K. Studies on the KKM-maps, MSRI-Korea, Rep. Ser., 14 (1985), 1-114.

PROLLA, J.B. Fixed point theorems for set valued mappings and the existence of best approximants, Numerical Functional Analysis and Optimization, 5 (4) (1982-83), 449-455.

SEIIGAL, V.M., SINGII, S.P., AND GASTL, , G.C. On a fixed point theorem for multivalued maps, Proc. First Intern. Conf. on Fixed Point Theory, Pitman Publishers, London, U.K. (1991), 377-382. 


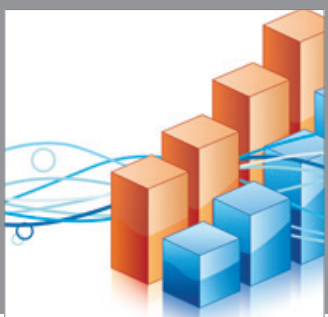

Advances in

Operations Research

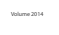

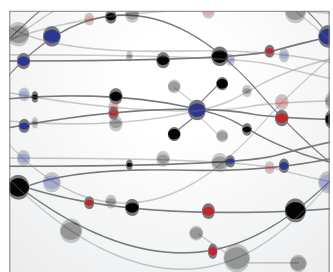

\section{The Scientific} World Journal
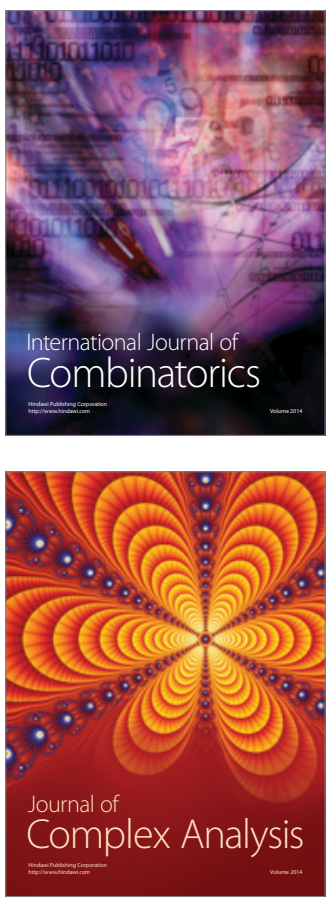

International Journal of

Mathematics and

Mathematical

Sciences
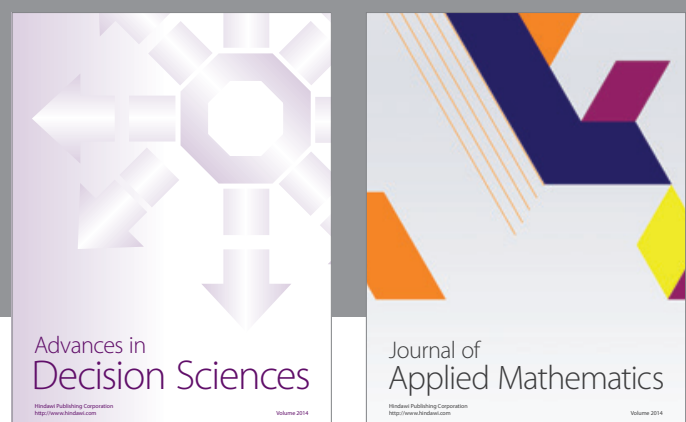

Journal of

Applied Mathematics
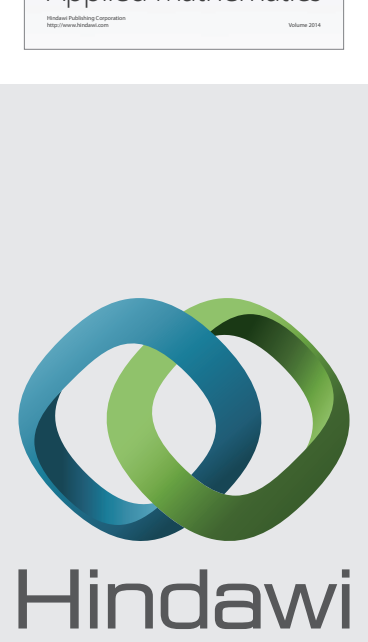

Submit your manuscripts at http://www.hindawi.com
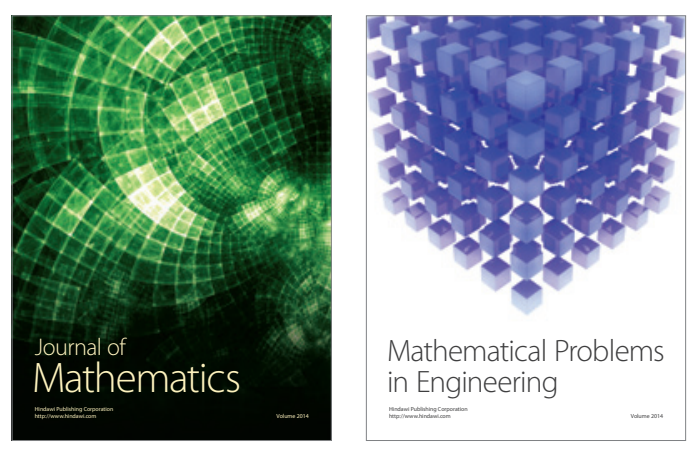

Mathematical Problems in Engineering
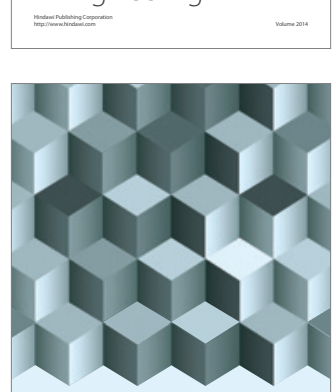

Journal of

Function Spaces
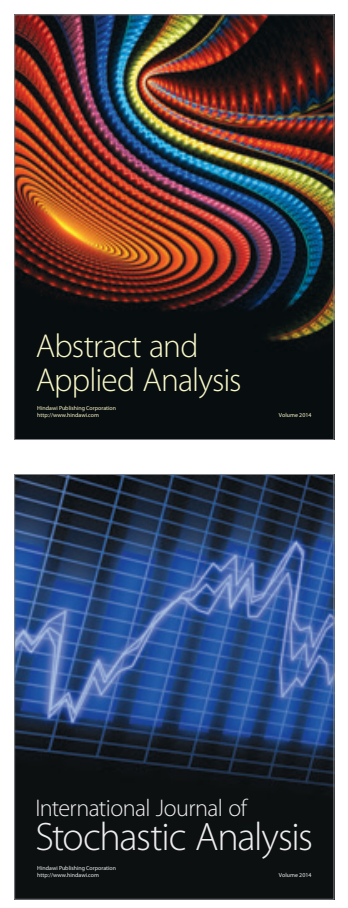

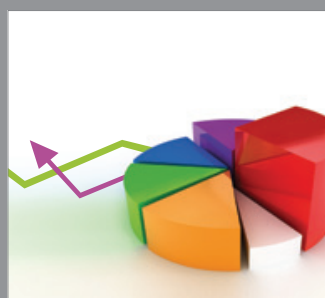

ournal of

Probability and Statistics

Promensencen
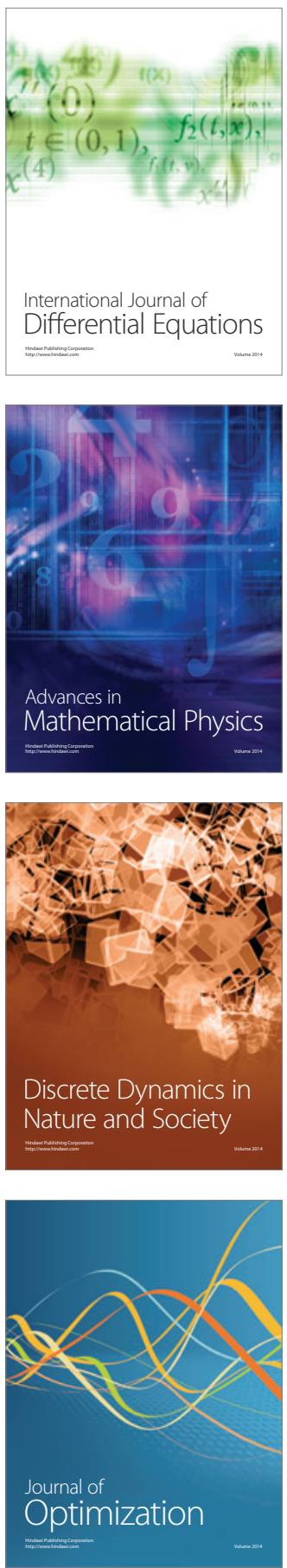